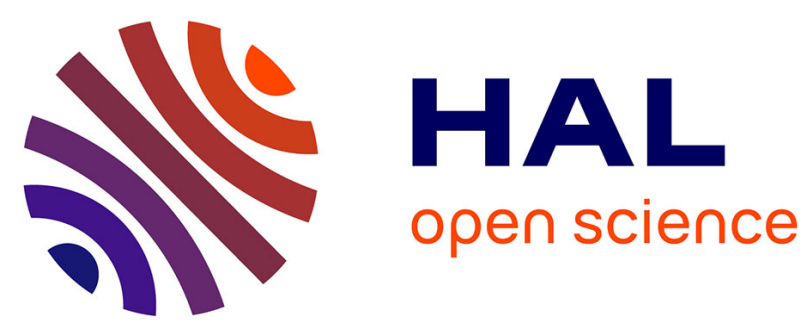

\title{
Transport and self-organization in dissipative drift-wave turbulence
}

Thierry Dudok de Wit, V. Krasnosel'Skikh, L. Wooliscroft, M. Balikhin, S. Bale, M. Dunlop, H. Lühr, S. Schwartz, L. Woolliscroft, S. Benkadda, et al.

\section{To cite this version:}

Thierry Dudok de Wit, V. Krasnosel'Skikh, L. Wooliscroft, M. Balikhin, S. Bale, et al.. Transport and self-organization in dissipative drift-wave turbulence. Physical Review E , 1995, 52 (6), pp.6753-6758. 10.1103/PhysRevE.52.6753 . insu-03388664

\section{HAL Id: insu-03388664 https://hal-insu.archives-ouvertes.fr/insu-03388664}

Submitted on 20 Oct 2021

HAL is a multi-disciplinary open access archive for the deposit and dissemination of scientific research documents, whether they are published or not. The documents may come from teaching and research institutions in France or abroad, or from public or private research centers.
L'archive ouverte pluridisciplinaire HAL, est destinée au dépôt et à la diffusion de documents scientifiques de niveau recherche, publiés ou non, émanant des établissements d'enseignement et de recherche français ou étrangers, des laboratoires publics ou privés. 


\title{
Transport and self-organization in dissipative drift-wave turbulence
}

\author{
T. Dudok de Wit, ${ }^{*}$ S. Benkadda, P. Gabbai, and A.D. Verga \\ Equipe Turbulence Plasma, CNRS, ${ }^{\dagger}$ Institut Méditerranéen de Technologie, \\ F-13451 Marseille Cedex 20, France
}

(Received 16 May 1995)

\begin{abstract}
The contribution of coherent structures to steady-state transport in dissipative drift-wave turbulence is investigated. Using the two-field Hasegawa-Wakatani model as a paradigm for studying self-organization in magnetized plasmas, we show in an unambiguous manner that coherent vortex structures play a dominant role in cross-field transport.
\end{abstract}

PACS number(s): 52.35.Ra, 47.27.-i, 02.50.Wp

\section{INTRODUCTION}

One of the most fascinating aspects of fully developed turbulence, as it is observed in two-dimensional (2D) fluids or plasmas, is the emergence of coherent vortex structures $[1,2]$. Such structures are believed to play a considerable role on the macroscopic properties of the system, in particular the steady-state transport of particles and energy. The basic understanding of their dynamics is not yet well understood, due in part to the difficulty in identifying them from the experimental or computational signals. The identification of the coherent structures from spatiotemporal turbulence measurements has been a challenging problem so far due to the lack of adequate analysis techniques [3]. While it is generally easy to detect coherent structures by eye, it is much more difficult to identify and extract them in an objective way. As a consequence, most of our knowledge remains based on flow visualization [2] or averaging techniques [5] and little quantitative evidence has been obtained so far for a link between transport and self-organization. Recently, using a multivariate technique called the biorthogonal decomposition (BOD), we were able to characterize some properties of coherent structures observed in experimental data issued from edge tokamak measurements [4].

In the present paper, we address the issue of the influence of coherent structures on the (particle) transport, in the context of generic dissipative drift wave turbulence using the two-field density and electrostatic potential fluctuations, Hasegawa-Wakatani (HW) model [6]. This model is well known to exhibit both an adiabatic regime, when the electrons have a Boltzmann distribution, and a hydrodynamic regime, where electrons and ions act essentially as a passive scalar. This model pro-

\footnotetext{
*Present address: Laboratoire de Physique et Chimie de l'Environnement, CNRS, 3A, Avenue de la Recherche Scientifique, F-45071 Orléans Cedex 2, France.

${ }^{\dagger}$ URA 773 du Centre National de la Recherche Scientifique et de l'Université de Provence.
}

$1063-651 X / 95 / 52(6) / 6753(6) / \$ 06.00$ vides a paradigm for studying cross-field transport in the presence of coherent vortex structures and bears a close resemblance to the turbulence observed in the ionosphere [7] and in tokamak edge plasmas [8]. This model, which neglects temperature fluctuations, magnetic shear and toroidal geometry, does not pretend to describe tokamak turbulence [9]. It contains, however, the basic elements we want to investigate in connection to the transport: a large spectrum of turbulent fluctuations and the formation of coherent structures. This model is well suited for testing nonstandard analysis techniques of spatiotemporal signals.

Using two complementary analysis techniques, the BOD and the conditional averaging, we show that (i) the impact of coherent structures on cross-field transport can be quantified and (ii) the transport properties in the dissipative drift wave turbulence strongly deviate from the usual quasilinear regime due to the influence of large-scale and long-lived vortex structures. This paper is organized as follows. In Sec. II the simulation data of density, potential, and radial flux of the HW system are presented. In Sec. III the flux is analyzed with a proper orthogonal decomposition method and with the conditional averaging. Section IV is devoted to the conclusion.

\section{SIMULATION DATA}

The two-field HW model provides a set of nonlinear coupled equations for electron density $n$ and electrostatic potential $\phi$ fluctuations in an inhomogeneous magnetized plasma

$$
\begin{gathered}
\left(\frac{\partial}{\partial t}-D \Delta\right) n+\frac{\partial}{\partial y} \phi+c(n-\phi)=[n, \phi] \\
\left(\frac{\partial}{\partial t}-\nu \Delta\right) \Delta \phi+c(n-\phi)=[\Delta \phi, \phi] .
\end{gathered}
$$

Time is given in units $L_{n} / c_{s}\left(L_{n}\right.$ is the gradient density scale length and $c_{s}$ the acoustic speed), space is in hy- 

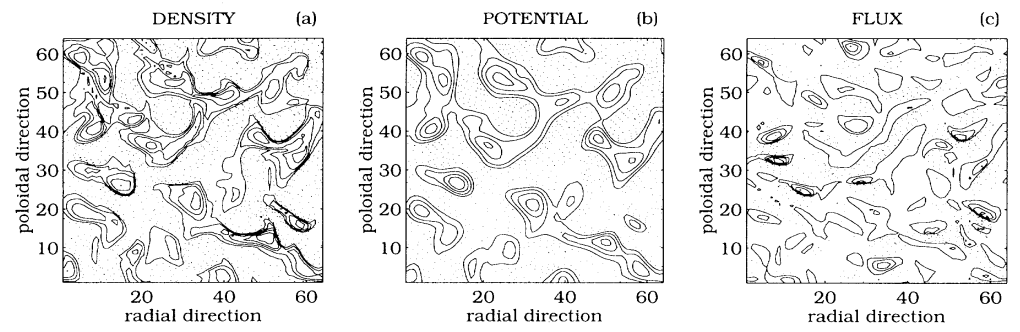

FIG. 1. Contour plots of the (a) density, (b) potential, and (c) flux fluctuations levels, at a given time. Dotted lines refer to negative amplitudes.

brid gyroradii $\rho_{s}, c$ is the adiabaticity parameter, $\nu$ is the viscosity, $D$ is the diffusion coefficient, $x$ and $y$ stand for the radial and poloidal coordinates, respectively, and [, ] denotes Poisson brackets. The fundamental parameter in these equations is $c$, which takes into account the parallel current and controls the response of the electrons. In the limit $c \rightarrow 0$ the density equation is decoupled from the potential one and the system is in an hydrodynamic regime: the potential equation (2.2) reduces to the usual Euler equation of two-dimensional flows: in the $c \rightarrow \infty$ limit, the density follows the potential and then the system is in the adiabatic regime: the two equations reduce to the Hasegawa-Mima model.

These equations were solved using a pseudospectral method (discussed in [10]) on a $128 \times 128$ periodic grid of dimensions $64 \rho_{s}$, which extends both poloidally and radially. Typical parameters used are $c=0.7, \nu=0.01$, and $D=0.01$. For this value of $c$, the system is in an intermediate regime, ensuring a growth of unstable modes and allowing the existence of a net transport, without being completely hydrodynamical. For the purposes of this work the spatial resolution of the simulation is sufficient because we are essentially interested in the long and intermediate scales.

In the following, we consider an excerpt of 250 time periods obtained in the saturated state regime and with a spatial grid that has been decimated by 2 . Figure 1 shows 2D snapshots of the density and potential fluctuations and their associated flux defined by

$$
\Gamma=-n \frac{\partial \phi}{\partial y}
$$

where $\partial \phi / \partial y$ is the electric drift velocity in the radial direction. Large-scale structures are apparent, which evolve for several periods before they dissipate. The characteristic scale of these objects is of the order of $10 \rho_{s}$ and their liftime is about 10-20 turnover times. A visual inspection of the flux spatial distribution suggests a local enhancement in the vicinity of the coherent structures.

A mild level of intermittency is attested by slightly non-Gaussian probability distribution functions of the density and the potential (Fig. 2). On the other hand, the flux histogram [Fig. 2(c)] is consistent with a strong correlation between the density and the potential fluctuations, giving exponentially decaying tails (such as a $\chi^{2}$ distribution, since the flux is quadratic in the fluctuations).

We also compared the observed total flux with the quasilinear one, computed using the linear dispersion relation associated with Eqs. (2.1) and (2.2). We found that the flux observed in the saturated turbulent state is five times smaller than in the quasilinear case. This departure from the quasilinear flux may be explained by the presence of coherent structures. Similar investigations [2] have also indicated that transport may be associated with self-organization. In the following we make a more quantitative analysis of the influence of coherent structures on the transport.

\section{ANALYSIS OF THE FLUX}

The standard and simplest way to investigate the scales that are involved in the flux consists in analyzing the cross coherence

$$
\rho_{k_{y}}=\frac{\sum_{k_{x}, t_{n}} n_{k_{x}, k_{y}}\left(t_{n}\right) \phi_{k_{x}, k_{y}}^{*}\left(t_{n}\right)}{\sqrt{\sum_{k_{x}, t_{n}}\left|n_{k_{x}, k_{y}}\left(t_{n}\right)\right|^{2} \sum_{k_{x}, t_{n}}\left|\phi_{k_{x}, k_{y}}\left(t_{n}\right)\right|^{2}}}
$$
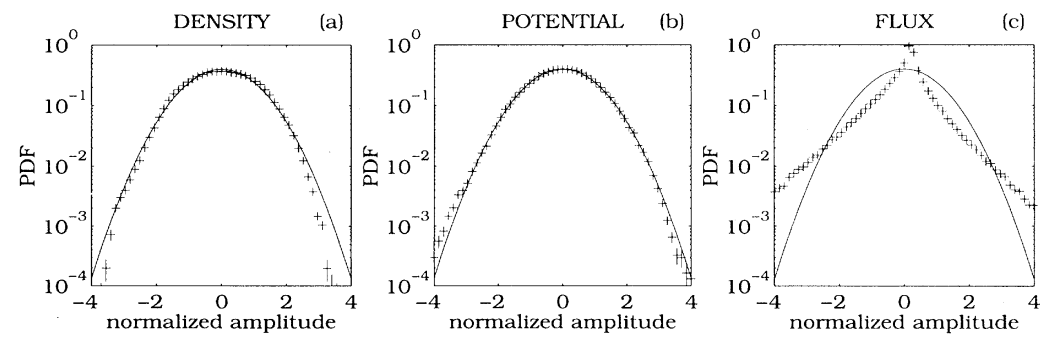

FIG. 2. Probability distribution functions associated with the (a) density, (b) potential fluctuations, and (c) flux. The quantities represented are centered at their mean values and normalized to their standard deviations. The inverted parabola corresponds to a reference Gaussian distribution with the same mean and standard deviation. 
and the phase shift between $n$ and $\phi$, defined by $\arg \left(\rho_{k_{y}}\right)$. The flux is maximum when the phase shift is $\pi / 2$ and the density and potential are well correlated. On the other hand, if the phases of $n$ and $\phi$ are random, the cross coherence vanishes and the flux also vanishes.

A strong coupling between these two quantities is attested by the large cross coherence; see Fig. 3. A correlation of density and potential is maintained across the whole spectrum. On the other hand, the phase shift between $n$ and $\phi$ is close to $\pi / 2$ for low (poloidal) wave numbers, but it is much smaller than the linear phase shift at higher wave numbers. In the nonlinear state the system sets a phase relation between the fluctuating fields that is almost inverted with respect to the linear estimation, where large $\mathbf{k}$ modes dominate. As a consequence of this relatively small contribution of the large wave numbers, the observed flux is weaker than the quasilinear estimation (obtained from the summation over the whole spectrum). This effect cannot be attributed to the adiabaticity parameter value or to the inverse cascade, which tends to concentrate the energy at large scales (the quasilinear computation uses the same value of $c$ and the same distribution of the potential, with its rapidly decaying power spectrum at high $\mathbf{k}$ ).

The $k_{y}$-power spectrum of the flux follows the phase shift pattern, then showing a maximum at intermediate wavelengths, approximatively at $10 \rho_{s}$. Although the coherent structure size is of the same order (see below), this is not a proof of a relation between self-organization and transport. Indeed, Fourier analysis is not suitable to a scale-length analysis and, moreover, this value of the wavelength coincides with the maximum growth rate of linear modes. In this sense an opposite conclusion may be

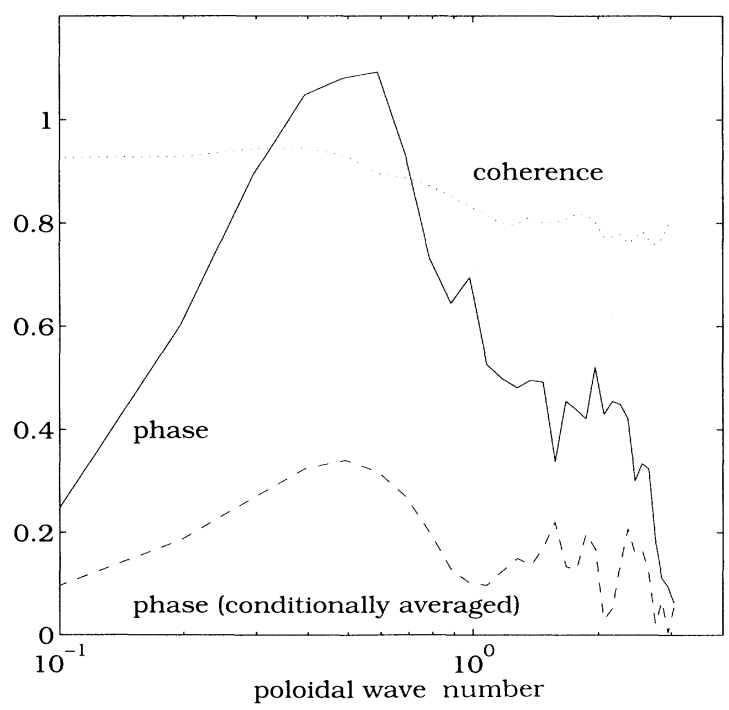

FIG. 3. Cross phase and cross coherence between the density and the potential vs the poloidal wave number. The dashed line represents the phase shift computed from the conditionally averaged density and potential fields. obtained: the absence of a strong link between large-scale organized structures and transport [11]. The interpretation of the cross spectrum, however, remains ambiguous as it cannot separate the effects of broadband turbulence and that of small structures convected by large ones. As will be shown now, the use of more appropriated analysis techniques can be more informative.

The first approach we consider is based on conditional averaging, which is a well-established technique for visualizing eddies in turbulence $[12,13]$. The heuristic idea is to add up ensembles of the data in such a way that random fluctuations average out but not organized structures. Figure 4(a) displays the time average of subsets of the density data whose amplitude level fit the constraint $n=2 \sigma_{n} \pm 5 \%$ at the center of the frame $\left(\sigma_{n}\right.$ being the standard deviation of $n$ ). A macroscopic pattern emerges, which can be considered as an average density eddy. Similar structures are obtained for the flux and the potential.

To study the relation between these eddies, we now perform a conditional analysis of the potential $\phi$ and the flux $\Gamma$, using the condition imposed on the density: each time a subset of $n$ is found to satisfy the condition, the same subset is taken for averaging from $\phi$ and $\Gamma$ [14]. We thus extract the average structures of the potential and the flux constrained to the sample obtained for the density in Fig. 4(a). The results, plotted in Figs. 4(b) and $4(\mathrm{c})$, reveal a structure in the potential whose phase shift to the density eddy is even smaller than the total phase shift shown in Fig. 3. Besides, Fig. 4(c) shows that the largest flux is also localized in the neighborhood of the coherent structure. We remark that this small shift between density and potential typical patterns is an indication of transport depletion by coherent structures relative to the quasilinear regime.

To assess this point, we computed the phase shift between the conditionally averaged density and potential; the result is represented in Fig. 3 and confirms that it is much smaller than the bare phase shift. This may suggest that indeed the transport is not simply a consequence of the vortex drift but is rather related to the complex structure of the fields and in particular to the smaller scales. At this stage, however, we are not able to quantify the contribution of the different scales to the transport. For this, a different technique is needed because the conditional averaging method omits the role of fluctuations to transport and smears out the scale richness of the fields.

The second approach we consider is based on the biorthogonal decomposition technique [15-17], which is also known as the proper orthogonal decomposition. This technique consists of decomposing the data into a finite set of orthonormal spatial and temporal components. The density fluctuation data, for example, are decomposed as

$$
n\left(x_{j}, t_{i}\right)=\sum_{m=1}^{250} A_{m} v_{m}\left(t_{i}\right) u_{m}\left(x_{j}\right),
$$

where for simplicity the two spatial coordinates are merged into one. This decomposition is unique [18] and the spatial and temporal components are respectively 

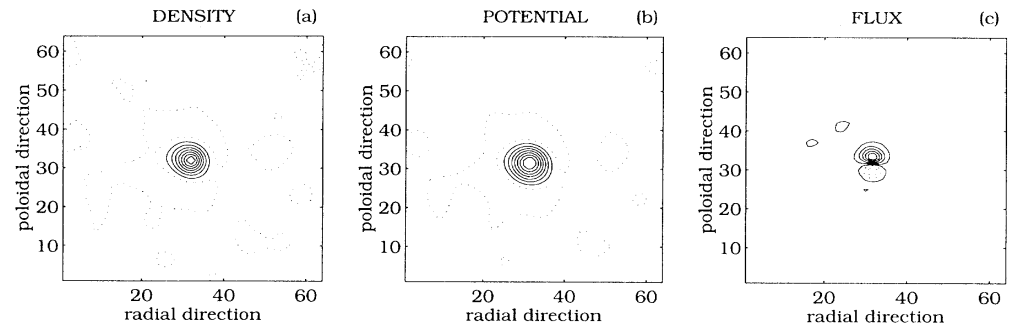

FIG. 4. Contour plots of the conditionally averaged (a) density, (b) potential, and (c) flux using the condition imposed on the density (843 samples are averaged).

eigensolutions of the time- and space-averaged data correlation matrices. The weights $A_{m}$ are either positive or zero and are conventionally sorted in decreasing order. The BOD has the interesting property to project large eddies on heavily weighted components whereas random fluctuations appear in modes that carry small weights. It thus separates fluctuation scales on the basis of their degree of correlation in space and in time.

The BOD of the fluctuation data leads to the weight distributions depicted in Fig. 5(a). The steepness of these distributions is an indication of redundancy and means that the salient features of the dynamics are captured by just a few terms out of the 250 . These distributions also show that the spatiotemporal behavior of the density is richer than that of the potential: more weights contribute significantly to the density field. The qualitative differences between density and potential are enhanced by diminishing the adiabaticity parameter $c$ : in the hydrodynamic limit the density behaves much like a passive scalar, with an added source term proportional to the poloidal component of the potential gradient [the $\partial \phi / \partial y$ term in (2.1)].

A spectral analysis of these terms [see Fig. 5(b)] shows that the characteristic poloidal $\left(\lambda_{y}=1 /\left\langle k_{y}\right\rangle\right)$ and temporal $(\tau=1 /\langle\omega\rangle)$ scales decrease with $m$ for large $m$ values [19]. For small $m$ values corresponding to the most significant modes in terms of energy content, we find definite length and time scales $\lambda_{y} \approx 10$ and $\tau \approx 20$, respectively. This is consistent with the conditional analysis and with the direct visualization of the field evolution. This result, together with the steepness of the weight distribution, is a clear signature of the existence of coherent structures [4]. Note that the characteristic poloidal length and the characteristic time associated with the intermediate and large $m$ modes follow the diffusive scaling $\lambda_{y} \approx \tau^{1 / 2}$, as expected for a random (Gaussian) field. This diffusion law does not hold for low $m$ modes associated with the coherent structures.

Another important point, revealed by the spectral analysis of the BOD modes, is that these modes are not monochromatic but contain a large spectrum of scales, $\lambda_{y}$ and $\tau$ being only the dominant ones. This means, by definition of the BOD, that large scale lengths are correlated with smaller ones. The coherent structure is not limited to the largest scales in the flow, but rather associated with its localized, long-lived component (at least in a statistical sense, as it is described by conditional analysis).

One interesting quantity to study is the poloidally averaged particle flux, given by $\Gamma_{r}=-\langle n \partial \phi / \partial y\rangle$; this quantity measures the transport across the magnetic field and is used, for instance, in edge tokamak transport studies. Although the BOD has essentially been used to reduce the dynamics of complex systems [20-22] to lowerdimensional spaces, it is equally appropriate for determining the contribution of the different scales to transport. To this end, we calculate the partial fluxes, defined as the fluxes carried by the normalized biorthogonal components of the density $\Gamma_{r}^{(m)}=-\left\langle n_{m} \partial \phi / \partial y\right\rangle$, where $n_{m}=v_{m} u_{m}$. The dependence of these partial fluxes on $\lambda_{y}$ is shown in Fig. 6 and reveals a strong correlation between the two quantities. This result confirms the role of self-organization on transport, which can now be quantified. Figure 6, for example, reveals that the impact of coherent structures is not due as much to some intrinsic property as to the fact that they have a larger spatiotemporal correlation.

In the view of these results, the mechanism of transport appears to be related to the advection of small (or intermediate-scale) fluctuations by the largest vortices. We see, in Fig. 6, that the flux grows with the scale
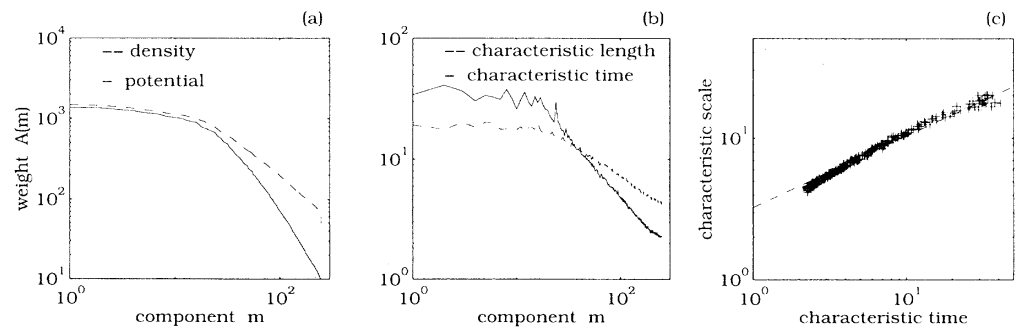

FIG. 5. Distribution of the biorthogonal weights of (a) the density and the potential, (b) characteristic time scales and poloidal scale lengths of the biorthogonal components of the density, (c) and log-log plot of the scale length vs the time scale of BOD modes. 


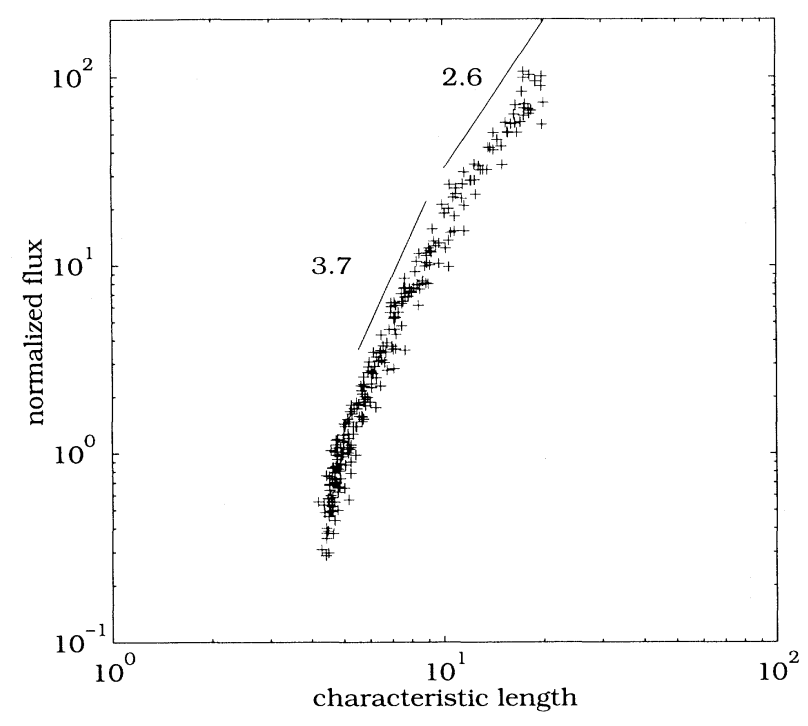

FIG. 6. Normalized partial fluxes associated with the biorthogonal components of the density vs their characteristic poloidal scale length. Note that the partial fluxes have been normalized vs the corresponding biorthogonal weights of the density so that large values truly reflect a significant contribution to transport.

as a power law with two different slopes depending on the scale. Although the existence of a knee in the data of Fig. 6 is clear, the values of the slopes are subject to uncertainties due to the few number of points used in the computation. We point out that this increase is not due to the fact that low-order modes are more heavily weighted, as the biorthogonal modes of the density have been normalized to their weight. Futhermore, no dependence of $\Gamma_{m}$ on $\lambda_{y}$ is observed when $n_{m}$ is replaced by Fourier modes having the same "scale length," thus indicating that low-order modes truly contribute to the transport.

This behavior may be explained by the fact that the normalized BOD mode density (the $n_{m}$ used in the computation of $\Gamma_{m}$ ) of the fluctuations in the neighborhood of the large vortex is proportional to $n_{m} \propto \lambda_{y}$ (the characteristic length of the vortex). The geometrical interpretation of the proportionality $n_{m} \propto \lambda_{y}$ for the intermediate scale lengths is that the fluctuations contributing to the transport are mainly located at the periphery of large vortices. Therefore, their normalized BOD mode density $\left(n_{m}\right)$ is not directly related to the area occupied by these fluctuations but to the length of the vortex which with they are correleted. Besides, these random fluctuations diffuse at a rate proportional to $v \propto \tau^{1 / 2}$, giving $\Gamma_{m} \propto \lambda_{y} \tau^{1 / 2} \approx \lambda_{y}^{2}$ (the large scale slope in Fig. 6). On the other hand, the smallest fluctuations, not at all correlated with the large vortices, contribute negligibly to the transport; the flux as a function of $\lambda_{y}$ decrease fast on these scales. For these scales the normalized density is proportional to the area of the fluctuations $n_{m} \propto \lambda_{y}^{2}$ (the
BOD weights decrease as $\lambda_{y}^{-2}$ ) and their velocity dispersion follows a ballistic law $v \propto \tau$. Therefore, at small scales we obtain $\Gamma_{m} \propto \lambda_{y}^{2} \tau \approx \lambda_{y}^{4}$ (the small scale slope in Fig. 6). We obtain in this way two different behaviors of the flux at small and large spatiotemporal scales, showing different power laws.

Let us note that this result is fundamentally different from what is expected from a simple mixing length ansatz, which will not provide the contribution of different scale lengths to the flux as it gives only a global value. Even if the mixing length, based on a random walk process, predicts a diffusion coefficient scaling as $k^{-2}$, this says nothing about the spatiotemporal dynamical behavior of the system. We found that this scaling is only relevant for intermediate scale lengths, fluctuating around larger vortices (themselves not contributing to the transport). Moreover, the BOD flux $\Gamma_{m}$ is completely different from the Fourier component $\Gamma_{k}$ of the flux, which enters in the mixing length theory. Indeed, the spatiotemporal BOD component takes into account a large spectrum of Fourier modes having the same correlation weight.

In summary, the picture that emerges here is that each coherent vortex is truly made out of a broad range of different spatial and temporal scales, in which the large ones predominate. These structures control the transport by the advection of intermediate-scale fluctuations.

\section{CONCLUSION}

In this paper we analyzed numerical simulations of the Hasegawa-Wakatani model to study the influence of selforganization on transport properties of the system. It has been clearly established that coherent vortex structures govern cross-field transport in dissipative drift-wave turbulence. In particular, the particle flux strongly increases with the size and the lifetime of the fluctuation events that carry it. From a comparison of different approaches (correlation and Fourier techniques, conditional analysis, and biorthogonal decomposition), a unified picture emerges, in which steady-state transport is related to the advection of intermediate-scale fluctuations at the steep gradients of the large structures. Coherent structures themselves are built from a large superposition of spatiotemporal scales and these scales satisfy a diffusionlike power law. We also found that radial flux obeys a power law in the characteristic scales. Two regimes of transport as a function of the characteristic spatiotemporal scales were identified and found to be related to two different power laws at small and large scales.

It must be pointed out that the existence of such vortices does not necessarily imply a significant deviation from Gaussianity, as the fluctuation probability distribution functions are found to be almost Gaussian.

Finally, we note that the conditional averaging and the BOD techniques were found to be appropriate in study- 
ing the spatiotemporal properties of a turbulent selforganized system. These techniques are not restricted to any particular model and a recent application of the latter to experimental data suggests that coherent structures also play a significant role in the transport of tokamak edge plasmas [4].

\section{ACKNOWLEDGMENTS}

One of us (T.D.) gratefully acknowledges the hospitality of the Equipe Turbulence Plasma. The simulations were performed on a Cray-YMP at the Centre Régional de Calcul Scientifique (Provence-Alpes-Côte d'Azur).
[1] J. McWilliams, J. Fluid Mech. 146, 21 (1984).

[2] A. Koniges, J. Crotinger, and P. Diamond, Phys. Fluids B 4, 2785 (1992).

[3] W. Horton, in Transport, Chaos and Plasma Physics, edited by S. Benkadda, F. Doveil, and Y. Elskens (World Scientific, Singapore, 1994), p. 200.

[4] S. Benkadda et al., Phys. Rev. Lett. 73, 3403 (1994).

[5] T. Huld, A. Nielsen, H. Pécseli, and J. Rasmussen, Phys. Rev. Lett. 64, 3023 (1990).

[6] A. Hasegawa and M. Wakatani, Phys. Fluids 27, 611 (1984).

[7] J. LaBelle, M. Kelley, and C. Seyler, J. Geophys. Res. 91, 5513 (1986).

[8] M. Endler et al. (unpublished).

[9] B. Scott, Phys. Fluids B 4, 2468 (1992).

[10] S. Benkadda, X. Garbet, and A. Verga, Contrib. Plasma Phys. 34, 247 (1994).

[11] D. Biskamp, S. Camargo, and B. Scott, Phys. Lett. A 186, 239 (1994).

[12] R. Antonia, Annu. Rev. Fluid Mech. 13, 131 (1981).

[13] H. Johnson, H. Pécseli, and J. Trulsen, Phys. Fluids 30,
2239 (1987).

[14] One could also impose the condition on $\phi$ or on $\Gamma$, or choose a different threshold level, but this does not significantly affect the conclusions.

[15] J. Lumley, Stochastic Tools in Turbulence (Academic, New York, 1970).

[16] N. Aubry, P. Holmes, J. Lumley, and E. Stone, J. Fluid Mech. 192, 192 (1988).

[17] G. Berkooz, P. Holmes, and J. Lumley, Annu. Rev. Fluid Mech. 25, 539 (1993).

[18] It is unique except when two or more weights are equal, in which case the components can be exchanged.

[19] The characteristic scales are defined as an average over the corresponding spectral power density $P$, i.e., $\langle\omega\rangle=$ $\int_{0}^{\omega_{\mathrm{Nyq}}} P(\omega) \omega d \omega / \int_{0}^{\omega_{\mathrm{Nyq}}} P(\omega) d \omega$, where the integration is carried up to the Nyquist limit.

[20] A. Newell, D. Rand, and D. Russell, Physica D 33, 281 (1988).

[21] L. Sirovich, Physica D 37, 126 (1989).

[22] T. Dudok de Wit, R. Lima, A. Pecquet, and J. Vallet, Phys. Plasmas 1, 3288 (1994). 